ALEA, Lat. Am. J. Probab. Math. Stat. 14, 733-749 (2017)

DOI: 10.30757/ALEA.v14-35

\title{
On the persistence regime for Lotka-Volterra in randomly fluctuating environments
}

\author{
Florent Malrieu and Pierre-André Zitt
}

Laboratoire de Mathématiques et Physique Théorique (UMR CNRS 7350),

Fédération Denis-Poisson (FR CNRS 2964),

Université François-Rabelais, Parc de Grandmont, 37200 Tours, France.

E-mail address: florent.malrieu@univ-tours.fr

Laboratoire d'Analyse et Mathématiques Appliquées (UMR CNRS 8050),

Université-Paris-Est-Marne-La-Vallée,

5, boulevard Descartes, Cité Descartes, Champs-sur-Marne,

77454 Marne-la-Vallée Cedex 2, France.

E-mail address: pierre-andre.zitt@u-pem.fr

\begin{abstract}
In this note, we study the long time behavior of Lotka-Volterra systems whose coefficients vary randomly. Benaïm and Lobry (2016) recently established that randomly switching between two environments that are both favorable to the same species may lead to different regimes: extinction of one species or the other, or persistence of both species. Our purpose here is to describe more accurately the range of parameters leading to these regimes, and the support of the invariant probability measure in case of persistence.
\end{abstract}

\section{Introduction}

1.1. The model. The aim of the present paper is to study the ergodicity of a piecewise deterministic Markov process (PDMP) linked to Lotka-Volterra type dynamics. These lines can be seen as a companion paper to Benaïm and Lobry (2016) since we go one step further in the description of different regimes of the process and the support of the invariant measures. Let us first provide an overview of the main results in Benaïm and Lobry (2016) before stating our contribution.

Received by the editors February 8th, 2016; accepted August 8th, 2017.

2010 Mathematics Subject Classification. 60J99, 34A60.

Key words and phrases. Population dynamics, persistence, piecewise deterministic processes, convex order, Markov processes.

Research supported by the French ANR grant ANR-12-JS01-0006-PIECE. 
For a given set of positive parameters $\mathcal{E}=(a, b, c, d, \alpha, \beta)$, consider the LotkaVolterra differential system in $\mathbb{R}_{+}^{2}$, given by

$$
\left\{\begin{array}{l}
\dot{x}=\alpha x(1-a x-b y) \\
\dot{y}=\beta y(1-c x-d y) \\
\left(x_{0}, y_{0}\right) \in \mathbb{R}_{+}^{2} .
\end{array}\right.
$$

This system modelizes the evolution of the populations of two species ( $x$ of type $\mathbf{x}$ and $y$ of type $\mathbf{y})$. The populations grow logistically - as encoded by the $\alpha x(1-a x)$ and $\beta y(1-d y)$ terms - and compete with each other, which gives rise to the cross terms $\alpha b x y$ and $\beta c x y$. We denote by $F_{\mathcal{E}}$ the associated vector field: $(\dot{x}, \dot{y})=$ $F_{\mathcal{E}}(x, y)$. In the sequel, the variable $z$ stands for $(x, y)$ and we will sometimes write $F_{\mathcal{E}}(z)$ instead of $F_{\mathcal{E}}(x, y)$. This ODE system, taken alone, is easy to analyze. In particular it has only a finite number of equilibrium points, towards which the dynamics converges. These equilibria may be on the coordinate axes - meaning that one of the species gets extinct - or inside the positive quadrant. The position and nature of the equilibria turn out to depend only on the signs of $c-a$ and $d-b$. A complete picture will be given in Section 2; let us note already that when $a<c$ and $b<d$, the point $(1 / a, 0)$ attracts any path starting in $(0,+\infty)^{2}$. We say in this case that the environnement is favorable to species $\boldsymbol{x}$; it leads to the extinction of species $\mathbf{y}$.

Consider now two such systems, labeled 0 and 1 , with environments $\mathcal{E}_{i}=$ $\left(a_{i}, b_{i}, c_{i}, d_{i}, \alpha_{i}, \beta_{i}\right)_{i=0,1}$. We make the following standing assumption:

Assumption 1.1. The environments $\mathcal{E}_{0}$ and $\mathcal{E}_{1}$ are both favorable to $\mathrm{x}: a_{i}<c_{i}$ and $b_{i}<d_{i}$ for $i=0,1$. In particular, taken alone, each system leads to the extinction of the second species, that is, $y_{t}$ converges to 0 and $\lim \sup x_{t}>0$ as soon as $x_{0}>0$.

Finally, introduce the random process obtained by switching between these two deterministic dynamics, at rates $\left(\lambda_{i}\right)_{i=0,1}$. More precisely, we consider the process $(Z, I)$ on $\mathbb{R}^{2} \times\{0,1\}$ driven by the infinitesimal generator

$$
L f(z, i)=F_{\mathcal{E}_{i}}(z) \cdot \nabla_{z} f(z, i)+\lambda_{i}(f(z, 1-i)-f(z, i)) .
$$

In other words, $I$ jumps from $i$ to $1-i$ after a random time with an exponential distribution of parameter $\lambda_{i}$, and while $I_{t}$ is equal to $i, Z$ evolves deterministically by $\dot{Z}_{t}=F_{\mathcal{E}_{i}}\left(Z_{t}\right)$. The coordinates of $Z_{t}$ are denoted by $X_{t}$ and $Y_{t}$. We refer to Benaïm and Lobry (2016) for a detailed biological motivation.

It is shown in Benaïm and Lobry (2016) that, depending on the environments $\mathcal{E}_{0}, \mathcal{E}_{1}$ and the jump rates $\lambda_{0}, \lambda_{1}$, one of the following four things occur almost surely:

- extinction of species $\mathbf{x}: X_{t} \rightarrow 0$ and $\lim \sup Y_{t}>0$,

- extinction of species $\mathbf{y}: Y_{t} \rightarrow 0$ and $\lim \sup X_{t}>0$,

- extinction of one of the two species, chosen randomly,

- persistence: the empirical occupation measure (and, in many cases, the distribution) of $\left(X_{t}, Y_{t}\right)_{t \geqslant 0}$ converges to a probability measure on $(0,+\infty)^{2}$ that is absolutely continuous with respect to the Lebesgue measure.

Moreover, one or more of these regimes may occur when the jump rates $\left(\lambda_{0}, \lambda_{1}\right)$ vary, the environments $\left(\mathcal{E}_{0}, \mathcal{E}_{1}\right)$ being fixed. Similar surprising behaviors for switched 
processes have been studied for linear ODEs in Benaïm et al. (2014); Lawley et al. (2014).

1.2. The frequent jumps asymptotics and the averaged vector field. Recall that $\lambda_{0}$, $\lambda_{1}$ are the jump rates from one environment to the other. Note that the index process $\left(I_{t}\right)_{t \geqslant 0}$ is Markov by itself, and its invariant measure is a Bernoulli distribution with parameter $\lambda_{0} /\left(\lambda_{0}+\lambda_{1}\right)$. As a consequence, it will be convenient to choose the alternative parametrization

$$
(s, t) \in[0,1] \times(0,+\infty) \mapsto(s t,(1-s) t)
$$

for the jump rates, that is, let $t$ be the sum $\lambda_{0}+\lambda_{1}$ and $s$ be the ratio $\lambda_{0} /\left(\lambda_{0}+\lambda_{1}\right)$.

Remark 1.2 (Length of interjump times I). Notice that the expectations of the interjump times are given by $(s t)^{-1}$ and $((1-s) t)^{-1}$. If $t$ is small, the jumps are rare and the jump times are large in average; as $t$ grows the jumps become more frequent and the jump times shorter on average.

As the parameter $t$ goes to infinity - the frequent jumps asymptotics - it can be shown that the stochastic process $\left(Z_{t}\right)_{t \geqslant 0}$ converges to the solution of

$$
\dot{z}_{t}=F_{s}\left(z_{t}\right) \quad \text { where } \quad F_{s}=(1-s) F_{\mathcal{E}_{0}}+s F_{\mathcal{E}_{1}} .
$$

As noticed in Benaïm and Lobry (2016), for any $s \in[0,1]$, the vector field $F_{s}$ is itself associated to a Lotka-Volterra system in an environment $\mathcal{E}_{s}=\left(a_{s}, b_{s}, c_{s}, d_{s}, \alpha_{s}, \beta_{s}\right)$, where

$$
\begin{array}{ll}
\alpha_{s}=(1-s) \alpha_{0}+s \alpha_{1}, & \beta_{s}=(1-s) \beta_{0}+s \beta_{1}, \\
a_{s}=\frac{(1-s) \alpha_{0} a_{0}+s \alpha_{1} a_{1}}{\alpha_{s}}, & b_{s}=\frac{(1-s) \alpha_{0} b_{0}+s \alpha_{1} b_{1}}{\alpha_{s}}, \\
c_{s}=\frac{(1-s) \beta_{0} c_{0}+s \beta_{1} c_{1}}{\beta_{s}}, & d_{s}=\frac{(1-s) \beta_{0} d_{0}+s \beta_{1} d_{1}}{\beta_{s}} .
\end{array}
$$

Recall that by our standing assumption, $a_{i}<c_{i}$ and $b_{i}<d_{i}$ for $i=0,1$. The key point is that these inequalities may be reversed for the averaged environment $\mathcal{E}_{s}$; in some situations $\mathcal{E}_{s}$ may even be unfavorable to species $\boldsymbol{x}$ !

Definition 1.3 (Critical parameter regions). Two critical (possibly empty) parameter regions are defined in Benaïm and Lobry (2016) by:

$$
I=\left(s_{1}, s_{2}\right)=\left\{s \in[0,1], a_{s}>c_{s}\right\}, \quad J=\left(s_{3}, s_{4}\right)=\left\{s \in[0,1], b_{s}>d_{s}\right\} .
$$

The fact that $I$ and $J$ are indeed intervals is obvious from the definition of $a_{s}$, $b_{s}, c_{s}$ and $d_{s}$ by (1.3). As we have seen, the relevance of these intervals stems from the fact that they correspond to different types for the averaged environment $\mathcal{E}_{s}$. For example, the vector field $F_{s}$ always has two stationary points on the coordinate axes, but their nature vary:

- the stationary point $\left(1 / a_{s}, 0\right)$ is a well if $s \notin I$ and a saddle point if $s \in I$,

- the stationary point $\left(0,1 / d_{s}\right)$ is a saddle point if $s \notin J$ and a well if $s \in J$.

Remark 1.4. Notice that if $a_{0}=a_{1}$ then the interval $I$ is empty. In the sequel we will focus on the case when $a_{0} \neq a_{1}$ and without loss of generality we will assume that $a_{0}<a_{1}$.

More details and a complete description of the phase portrait of the Lotka-Volterra system will be given below (see Section 2). 


\begin{tabular}{ccc}
\hline & $\Lambda_{\mathbf{y}}>0$ & $\Lambda_{\mathbf{y}}<0$ \\
\hline$\Lambda_{\mathbf{x}}>0$ & persistence of the two species & extinction of species $\mathbf{y}$ \\
\hline$\Lambda_{\mathbf{x}}<0$ & extinction of species $\mathbf{x}$ & Extinction of a randomly chosen species \\
\hline
\end{tabular}

TABle 1.1.

1.3. Invasion rates. It is shown in Benaim and Lobry (2016) that the regime of the process $(Z, I)$ is determined by the the signs of two quantities, called invasion rates, $\Lambda_{\mathbf{x}}$ and $\Lambda_{\mathbf{y}}$. These quantities are obtained by considering the system where one of the species, say $\mathbf{y}$, is almost extinct: $y \ll 1$. In this case, remark two things:

- if the current environment is $i$, the growth rate $\frac{\dot{y}}{y}$ of species $\mathbf{y}$ is approximately $\beta_{i}\left(1-c_{i} x\right)$, by $(1.1)$;

- while $y$ stays negligible, the evolution of $(X, I)$ looks like the dynamics of the Markov process on $[0, \infty) \times\{0,1\}$ driven by the generator

$$
L_{\mathbf{x}} f(x, i)=\alpha_{i} x\left(1-a_{i} x\right) \partial_{x} f(x, i)+\lambda_{i}(f(x, 1-i)-f(x, i)),
$$

which turns out to be ergodic with an invariant measure $\mu_{\mathbf{x}}$ on $[0, \infty) \times\{0,1\}$ that depends on the jump rates $\left(\lambda_{0}, \lambda_{1}\right)=(s t,(1-s) t)$.

Over a long period of time, the growth rate of $\mathbf{y}$ is therefore obtained by averaging $\beta_{i}\left(1-c_{i} x\right)$ with respect to the invariant measure $\mu_{\mathbf{x}}$ : this leads to the definition of the invasion rate $\Lambda_{\mathbf{y}}$ by

$$
\Lambda_{\mathbf{y}}(s, t)=\int_{[0, \infty) \times\{0,1\}} \beta_{i}\left(1-c_{i} x\right) \mu_{\mathbf{x}}(d x, d i) .
$$

When $\Lambda_{\mathbf{y}}$ is positive (respectively negative) species $\mathbf{y}$ tends to increase (respectively decrease) from low density. Similarly, one can define

$$
\Lambda_{\mathbf{x}}(s, t)=\int_{[0, \infty) \times\{0,1\}} \alpha_{i}\left(1-b_{i} y\right) \mu_{\mathbf{y}}(d y, d i) .
$$

where $\mu_{\mathbf{y}}$ is the invariant probability measure of the stochastic process on $\mathbb{R} \times\{0,1\}$ with generator

$$
L_{\mathbf{y}} f(y, i)=\beta_{i} y\left(1-d_{i} y\right) \partial_{y} f(y, i)+\lambda_{i}(f(y, 1-i)-f(y, i)),
$$

The main result in Benaim and Lobry (2016) ensures that the long time behavior of the Markov process $\left(Z_{t}, I_{t}\right)$ is characterized by the sign of these invasion rates, as summed up in the following table (see Theorems 3.1, 3.3, 3.4 and 4.1 of Benaïm and Lobry, 2016 for precise statements).

1.4. Our contribution. In view of the previous result, the study of the model is reduced to finding the sign of the invasion rates, depending on the parameters of the environment and on the jump rates. To state our results, we need to introduce a second parametrization for the jump rates $\left(\lambda_{0}, \lambda_{1}\right) \in(0,+\infty)^{2}$ slightly different from (1.2):

$$
(u, v) \in[0,1] \times(0,+\infty) \mapsto\left(\alpha_{0} u v, \alpha_{1}(1-u) v\right)
$$

in such a way that

$$
u=\gamma_{0} /\left(\gamma_{0}+\gamma_{1}\right) \quad \text { and } \quad v=\gamma_{0}+\gamma_{1} \quad \text { where } \gamma_{i}=\lambda_{i} / \alpha_{i} \quad \text { for } i=0,1 \text {. }
$$


The change of parameters $(u, v)=\xi(s, t)$ is triangular in the sense that $u$ only depends on $s$ :

$$
(u, v)=\xi(s, t)=\left(\frac{s \alpha_{1}}{(1-s) \alpha_{0}+s \alpha_{1}}, \frac{t}{\alpha_{0} \alpha_{1}}\left((1-s) \alpha_{0}+s \alpha_{1}\right)\right) .
$$

Remark 1.5 (Length of interjump times II). Notice that the new parameter $v$ is proportional to $t$ when $u$ (or $s$ ) is fixed. As a consequence, as in Remark 1.2, the interjump times are short when $v$ is large and large when $v$ is small.

Definition 1.6 (Reparametrized invasion rates). The invasion rates in the $(u, v)$ coordinates are denoted by

$$
\tilde{\Lambda}_{x}(u, v)=\Lambda_{x}\left(\xi^{-1}(u, v)\right) \quad \text { and } \quad \tilde{\Lambda}_{y}(u, v)=\Lambda_{y}\left(\xi^{-1}(u, v)\right) .
$$

Similarly, $\tilde{I}$ (resp. $\tilde{J}$ ) is the image of $I$ (resp. $J$ ) for the other parametrization.

Note that $\tilde{I}$ and $\tilde{J}$ still are (possibly empty) intervals.

Remark 1.7. The parameter $u$ is already implicitly considered in Benaïm and Lobry (2016), where it appears in the computations leading to the explicit conditions for the non-emptyness of $I$ (which are equivalent to the positivity of a second degree polynomial).

Our first result is an explicit formula for $\tilde{\Lambda}_{\mathbf{y}}$, suited both to fast numerical computations and theoretical study.

Lemma 1.8 (Expression of $\left.\tilde{\Lambda}_{\mathbf{y}}\right)$. Assume that $a_{0}<a_{1}$ (see Remark 1.4). Let $(u, v)$ given by (1.5). The quantity $\tilde{\Lambda}_{\mathbf{y}}$ can be rewritten as:

$$
\tilde{\Lambda}_{\mathbf{y}}(u, v)=\frac{1}{\left(a_{1}-a_{0}\right)\left(\frac{1}{\alpha_{0}}(1-u)+\frac{1}{\alpha_{1}} u\right)} \mathbb{E}\left[\phi\left(U_{u, v}\right)\right],
$$

where $\phi:[0,1] \rightarrow \mathbb{R}$ is defined by

$$
\phi(y)=\frac{1}{a_{0}+\left(a_{1}-a_{0}\right) y} P\left(a_{0}+\left(a_{1}-a_{0}\right) y\right)
$$

for some explicit second degree polynomial $P$, and the random variable $U_{u, v}$ follows a Beta distribution $\operatorname{Beta}(u v,(1-u) v)$.

Moreover, $\phi$ has the following properties:

- If $\tilde{I}=\emptyset$ then $\phi$ is nonpositive on $[0,1]$;

- If $\tilde{I}=\left(u_{1}, u_{2}\right) \neq \emptyset$ then $\phi$ is concave, negative on $\left(0, u_{1}\right) \cup\left(u_{2}, 1\right)$ and positive on $\tilde{I}$.

Recall that a function $f$ is called quasi convex on $(a, b)$ if its level sets $\{f \leqslant t\}$ are convex, that is, $f$ decreases on $(a, c)$ and increases on $(c, b)$ for some $c \in[a, b]$.

Our main result describes more precisely the region of positivity for $\Lambda_{\mathbf{x}}$ and $\Lambda_{\mathbf{y}}$.

Theorem 1.9 (Shape of the regions). There exists a function $u \mapsto v_{\mathbf{y}}(u)$ from $(0,1) \rightarrow[0, \infty]$, with domain $\tilde{I}$, such that $\tilde{\Lambda}_{\mathbf{y}}(u, v)<0$ when $v<v_{\mathbf{y}}(u)$ and $\tilde{\Lambda}_{\mathbf{y}}(u, v)>0$ when $v>v_{\mathbf{y}}(u)$.

Moreover $v_{\mathbf{y}}$ is quasi-convex, continuous on its domain $\tilde{I}$, and tends to $+\infty$ on the endpoints of $\tilde{I}$.

Similarly, there exists a function $s \mapsto t_{\mathbf{y}}(s) \in[0, \infty]$, with domain $I$, going to infinity at the endpoints of $I$, such that: 
- $\Lambda_{\mathbf{y}}(s, t)<0$ if $t<t_{\mathbf{y}(\mathrm{s})}$,

- $\Lambda_{\mathbf{y}}(s, t)=0$ if $t=t_{\mathbf{y}(\mathrm{s})}$,

- $\Lambda_{\mathbf{y}}(s, t)>0$ if $t>t_{\mathbf{y}(\mathrm{s})}$.

The same statement holds in the parameters $(s, t)$ for the function $\left(-\Lambda_{\mathbf{x}}\right)$ with $I$ replaced by $J$ and with a critical function $t_{\mathbf{x}}(s)$.

Remark 1.10. Numerical computations suggest that both $v_{y}$ and $t_{y}$ are in fact smooth and convex on $\tilde{I}$ and $I$ respectively.

Remark 1.11. This result is cited in Benaïm and Lobry (2016, Proposition 2.5), since it answers a conjecture that appeared in a preprint version of Benaïm and Lobry (2016).

For an illustration of Theorem 1.9 and Remark 1.10, see Figure 1.1.

Finally, our last results are dedicated to the support of the non-trivial invariant probability measure in the persistence regime. In Benaïm and Lobry (2016), it is shown that this measure has a density with respect to the Lebesgue measure on the quadrant. Theorem 5.1 provides a full description of its support when the set $I \cap J$ is not empty. Since a precise statement requires several notations introduced in Section 2, we postpone it to the last section of the document.

The remainder of the paper is organized as follows. In Section 2 we describe the various phase portraits for Lotka-Volterra vector fields, and narrow down the choices of $\mathcal{E}_{0}, \mathcal{E}_{1}$ that lead to interesting behaviour. In Section 3 we prove Lemma 1.8; the main result is proved in Section 4. The final section is dedicated to the description of the support of invariant measures in the persistence regime.

\section{Deterministic picture}

2.1. Phase portraits of Lotka-Volterra vector field. We consider here the ODE (1.1) in an environment $\mathcal{E}=(a, b, c, d, \alpha, \beta)$ and describe its possible qualitative behaviours. Much of this description can be found in Benaïm and Lobry (2016), we give it here for the sake of clarity.

Barring limit cases that we will not consider, there are essentially four different phase portraits for the system, that are depicted in Figure 2.2. These four regimes are obtained as follows.

Notice first that the vector $F_{\mathcal{E}}(x, y)$ is horizontal if $y=0$ or $c x+d y=1$ : we call the line $c x+d y=1$ the horizontal isocline. Similarly $F_{\mathcal{E}}(x, y)$ is vertical if $x=0$ or if $(x, y)$ is on the vertical isocline $a x+b y=1$. These isoclines are the bold straight lines in Figure 2.2.

Each axis is invariant that why in the sequel we are only interested in initial conditions with positive coordinates. The three points $(0,0),(0,1 / d)$ and $(1 / a, 0)$ are stationary for $F_{\mathcal{E}}$. The origin is always a source. The nature of the other points and the existence of a fourth stationary point depends on the parameters; this gives rise to the four types announced above.

Type 1. If $a<c$ and $b<d$, species $\mathbf{y}$ gets extinct.

- The point $(1 / a, 0)$ is the unique global attractor: any solution of the ODE starting from a point with positive coordinates converges to $(1 / a, 0)$.

- The point $(0,1 / d)$ is a hyperbolic saddle point. Its stable manifold is the vertical axis. We denote by $\Sigma$ the intersection of its unstable manifold with 

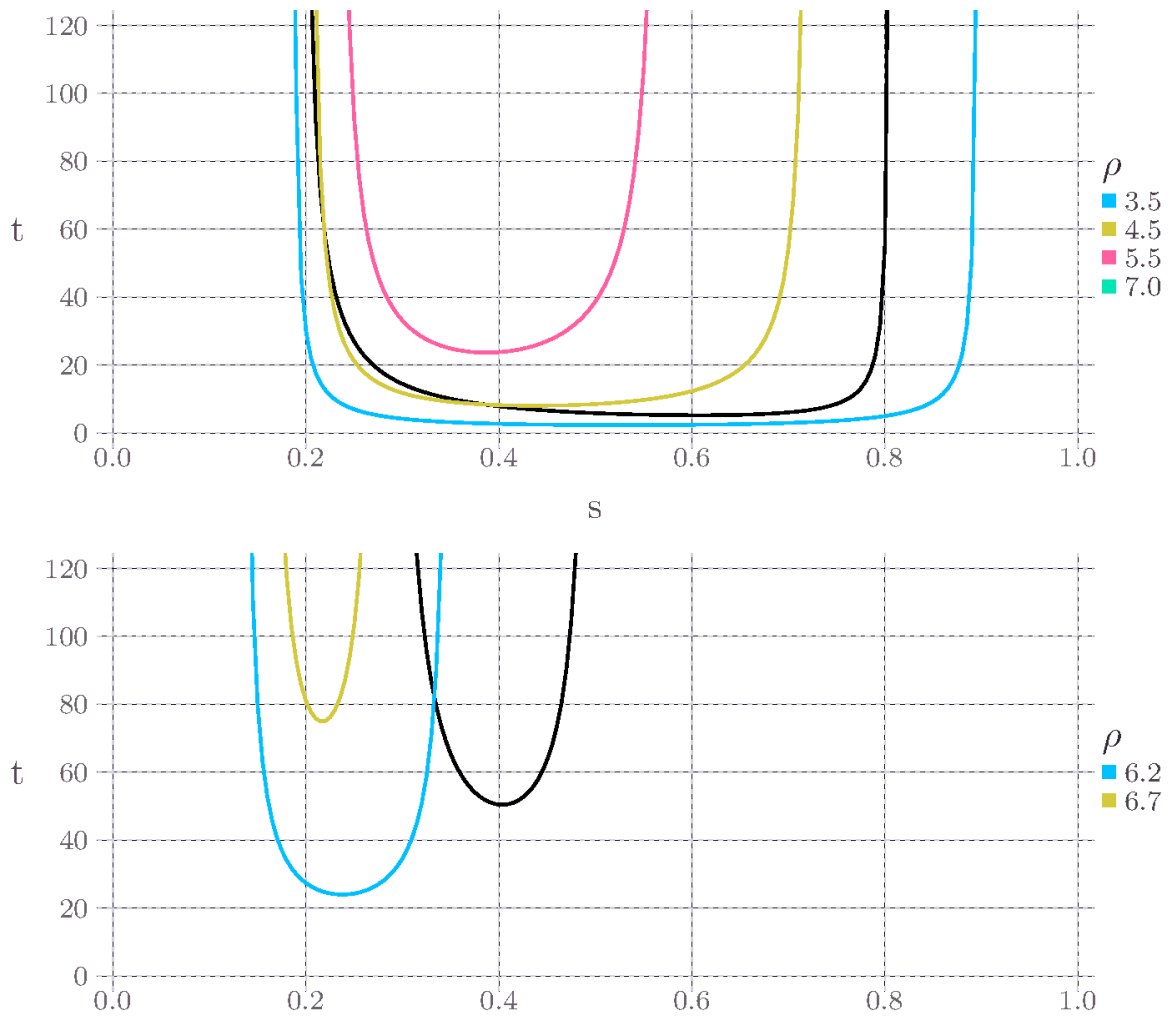

These plots represent the "critical" functions $t_{\mathbf{y}}$ and $t_{\mathbf{x}}$ for different choices of the environments. Denoting environments by the couple $\left(\begin{array}{c}\alpha \\ \beta\end{array}\right) ;\left(\begin{array}{ll}a & b \\ c & d\end{array}\right)$, the functions are plotted with

$\mathcal{E}_{0}=\left(\begin{array}{l}1 \\ 5\end{array}\right) ;\left(\begin{array}{ll}1 & 1 \\ 2 & 2\end{array}\right)$ (top plot) or $\left(\begin{array}{l}1 \\ 2\end{array}\right) ;\left(\begin{array}{ll}1 & 2 / 3 \\ 2 & 4 / 3\end{array}\right)$ (bottom plot); $\mathcal{E}_{1}=\left(\begin{array}{l}5 \\ 1\end{array}\right) ;\left(\begin{array}{ll}3 & 3 \\ 4 & \rho\end{array}\right)$

for various values of the parameter $\rho$ appearing in the definition of the environment $\mathcal{E}_{1}$. The black curve in both plots is $t_{\mathbf{y}}$, and does not depend on the value of $\rho$. The colored curves are $t_{\mathbf{x}}$. The respective domains of these curves are the intervals $I$ and $J$. All configurations are possible: $I \cap J$ may be empty (bottom plot, $\rho=6.8$ ), a strict subset of $I$ and $J$ (bottom plot, $\rho=6.2$ ) or may be $I$ or $J$ itself (top plot). Thanks to the results of Benaïm and Lobry (2016) summarized in the Table 1.1, these plots describe exactly what regimes are possible when the jump rates (parametrized by $s$ and $t$ ) vary, for a given choice of the environments. For example the top plot for $\rho=5.5$ has three regimes: extinction of $\mathbf{x}$ (above the red curve), persistence (between the red and the black curves) and extinction of $\mathbf{y}$ (below the black curve). For $\rho=4.5$ there is an additional zone (above the yellow curve and below the black one) of extinction of a random species. In particular, the knowledge of the relative positions of $I$ and $J$ is not enough to determine the possible regimes. All these plots are computed by finding, for a fixed $s$, the zero of the function $t \mapsto \Lambda(s, t)$; this is done by a simple root finding algorithm, using the explicit formula given in Lemma 1.8 to evaluate $\Lambda(s, t)$.

FiguRE 1.1. Shape of positivity regions for $\Lambda_{\mathbf{x}}$ and $\Lambda_{\mathbf{y}}$ 
the positive quadrant: $\Sigma$ is the curve made of points $\left(x_{0}, y_{0}\right)$ such that the solution $\left(x_{t}, y_{t}\right)$ starting at $\left(x_{0}, y_{0}\right)$ satisfies

$$
\left(x_{t}, y_{t}\right) \underset{t \rightarrow-\infty}{\longrightarrow}(0,1 / d) \text {. }
$$

We may compute explicitly some characteristics of $\Sigma$ : in particular, it leaves $(0,1 / d)$ with a slope $-\frac{\alpha(d-b)+\beta d}{\beta c}$ and ends in $(1 / a, 0)$ with a possibly degenerate slope $\min \left(0,-\frac{\beta(a-c)+\alpha a}{\alpha b}\right)$. Moreover, $\Sigma$ lies in between the horizontal and vertical isoclines.

Type 2. If $a>c$ and $b>d$, species $\mathbf{x}$ gets extinct. $(0,1 / d)$ is the unique sink and $(1 / a, 0)$ is a saddle point. This is the same as Type 1 except that the two species $\mathbf{x}$ and $\mathbf{y}$ are swapped.

Type 3. If $a>c$ and $b>d$, both species survive. The points $(1 / a, 0)$ and $(0,1 / d)$ are saddle points. The isoclines meet at the $\operatorname{sink}(\bar{x}, \bar{y})=(a d-b c)^{-1}(a-c, b-d)$, which is the unique global attractor.

Type 4. If $a>c$ and $b<d$, one of the two species gets extinct depending on the starting point. The meeting point of the isoclines $(\bar{x}, \bar{y})=(a d-b c)^{-1}(a-c, d-b)$ is now a saddle point, and (non trivial) trajectories converge to one of the two sinks on the axes, $(1 / a, 0)$ and $(0,1 / d)$.

2.2. Relative positions of the two environments. Recall that the vector fields $F_{\mathcal{E}_{0}}$ and $F_{\mathcal{E}_{1}}$ are assumed favorable to species $\mathbf{x}: a_{i}<c_{i}$ and $b_{i}<d_{i}$ for $i=0,1$. Without loss of generality, we suppose that $a_{0}<a_{1}$. The switched system may present a surprising behavior if the interval $I$ defined in Equation (1.4) is not empty. This requires that $c_{0}<a_{1}$. As a consequence, we impose in the sequel that

$$
a_{0}<c_{0}<a_{1}<c_{1}, \quad b_{0}<d_{0} \quad \text { and } \quad b_{1}<d_{1} .
$$

Lemma 2.1. If the set $J$ is not empty then $d_{1}<b_{0}$ or $d_{0}<b_{1}$. Moreover, if $I \cap J$ is non empty then

$$
a_{0}<c_{0}<a_{1}<c_{1} \quad \text { and } \quad b_{0}<d_{0}<b_{1}<d_{1} .
$$

Proof: The first point is clear from the definition of $J$. As a consequence, two configurations are compatible with $I$ and $J$ non empty: (2.1) and

$$
a_{0}<c_{0}<a_{1}<c_{1} \text { and } b_{1}<d_{1}<b_{0}<d_{0}
$$

Suppose, to derive a contradiction, that we are in the latter configuration. Define the points $C_{i}=\left(1 / c_{i}, 0\right)$ and $D_{i}=\left(0,1 / d_{i}\right)$ for $i=0,1$. If $M=(\tilde{x}, \tilde{y})$ is the intersection of the lines $\left(C_{0} D_{0}\right)$ and $\left(C_{1} D_{1}\right)$ then the set $[\tilde{x},+\infty) \times[0, \tilde{y}]$ is strongly positively invariant under the action by $F_{0}$ and $F_{1}$. Furthermore, if $I \cap J$ is not empty, this means that there exists $s_{0} \in(0,1)$ such that $F_{\mathcal{E}_{s_{0}}}$ is of Type 2. In particular, the point $\left(0,1 / d_{s}\right)$ is accessible. This is incompatible with the previous remark. As a consequence, $I \cap J$ is empty for this configuration.

\section{Expression of the invasion rate}

Let us first recall the expression of $\Lambda_{\mathbf{y}}$ derived in Benaïm and Lobry (2016). Letting $p_{i}=1 / a_{i}, \Lambda_{\mathbf{y}}$ is given by

$$
\Lambda_{\mathbf{y}}=p_{0} p_{1} C \int_{p_{0}}^{p_{1}} P(x) \theta(x) d x
$$



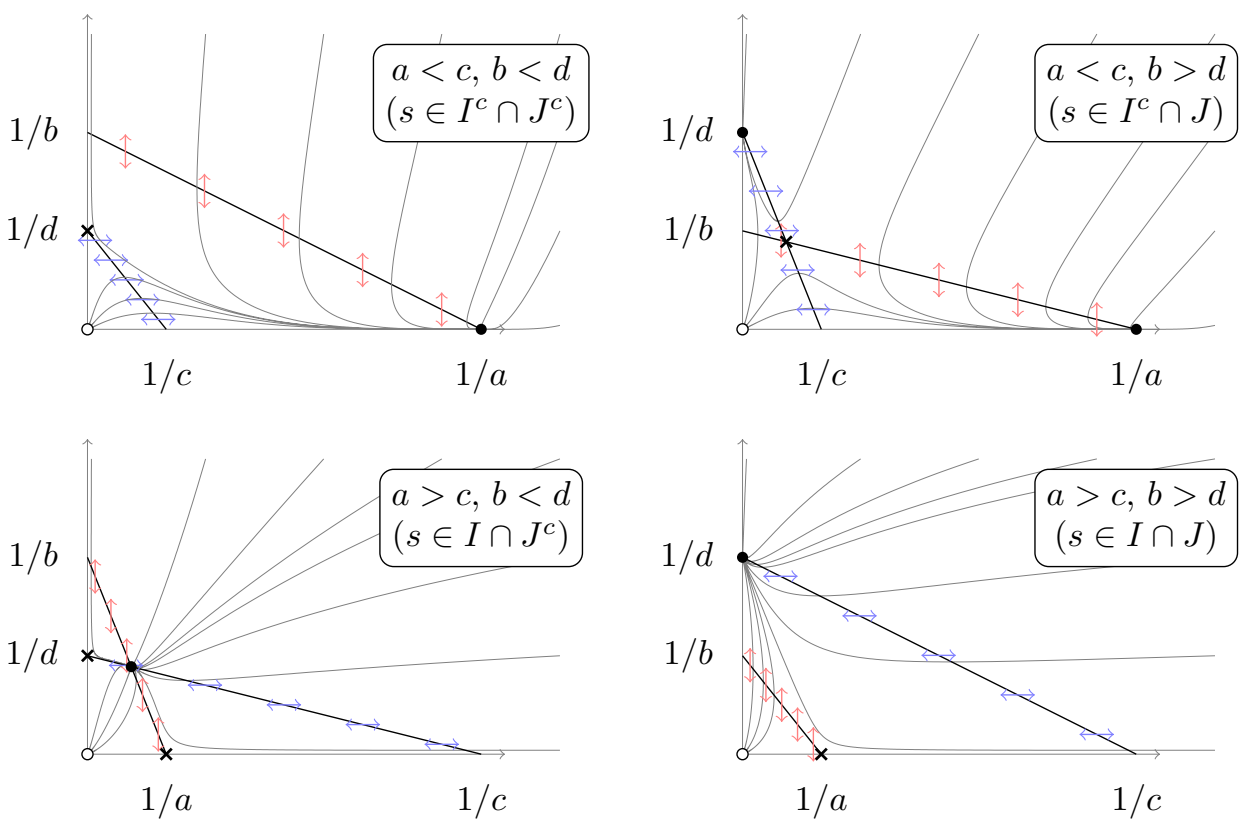

In each picture the bold lines are the horizontal and vertical isoclines, the gray lines are trajectories of the ODE. Sources, sinks and saddle points are pictured respectively by white circles, black circles and crosses. Type 1 environments correspond to the upper left picture, Type 2 to the bottom right one. In Type 3 environments (bottom left), the intersection of the isoclines attracts the whole quadrant. In Type 4 environments (upper right) one species or the other gets extinct depending on the starting point. For fixed environments $\mathcal{E}_{0}, \mathcal{E}_{1}$ of Type 1 , the mixed environment $\mathcal{E}_{s}$ may be of any of the four types, depending on whether $s$ belongs to the intervals $I$ and $J$.

Figure 2.2. The four possible deterministic regimes for a given environment

where

$$
\begin{aligned}
C^{-1}= & \frac{p_{1}}{\alpha_{1}} \int_{\min \left(p_{0}, p_{1}\right)}^{\max \left(p_{0}, p_{1}\right)} \frac{\left|x-p_{0}\right|^{\gamma_{0}}\left|p_{1}-x\right|^{\gamma_{1}-1}}{x^{\gamma_{0}+\gamma_{1}+1}} d x \\
& +\frac{p_{0}}{\alpha_{0}} \int_{\min \left(p_{0}, p_{1}\right)}^{\max \left(p_{0}, p_{1}\right)} \frac{\left|x-p_{0}\right|^{\gamma_{0}-1}\left|p_{1}-x\right|^{\gamma_{1}}}{x^{\gamma_{0}+\gamma_{1}+1}} d x, \\
\theta(x)= & \frac{\left|x-p_{0}\right|^{\gamma_{0}-1}\left|p_{1}-x\right|^{\gamma_{1}-1}}{x^{\gamma_{0}+\gamma_{1}+1}}, \\
P(x)= & {\left[\frac{\beta_{1}}{\alpha_{1}}\left(1-c_{1} x\right)\left(1-a_{0} x\right)-\frac{\beta_{0}}{\alpha_{0}}\left(1-c_{0} x\right)\left(1-a_{1} x\right)\right] \frac{a_{1}-a_{0}}{\left|a_{1}-a_{0}\right|} . }
\end{aligned}
$$

This quantity is obtained by averaging the growth rate of the second species with respect to the invariant measure of the one-dimensional $\operatorname{PDMP}(X, I)$ on $[0, \infty) \times$ $\{0,1\}$ driven by

$$
L f(x, i)=\alpha_{i} x\left(1-a_{i} x\right) \partial_{x} f(x, i)+\lambda_{i}(f(x, 1-i)-f(x, i)),
$$


which corresponds to the dynamics of species $\mathbf{x}$ when species $\mathbf{y}$ is gone. In the sequel, we assume that $a_{0}<a_{1}$ and set $\delta=a_{1}-a_{0}$. It is obvious that the recurrent set of $(X, I)$ is $\left[p_{1}, p_{0}\right] \times\{0,1\}$.

In Benaïm and Lobry (2016), the continuous part $\left[p_{1}, p_{0}\right]$ of the state space is parametrized by a mapping $[0,1] \ni s \mapsto p_{s}$ given by $p_{s}=1 / a_{s}$, where $a_{s}=\left(s \alpha_{1} a_{1}+\right.$ $\left.(1-s) \alpha_{0} a_{0}\right) /\left(s \alpha_{1}+(1-s) \alpha_{0}\right)$. It is interesting to look at another parametrization $[0,1] \ni u \mapsto \tilde{p}(u)$, where $\tilde{p}(u)=\tilde{a}(u)^{-1}=\frac{1}{a_{0}+\delta u}$. These parametrizations are summed up in the following diagram:

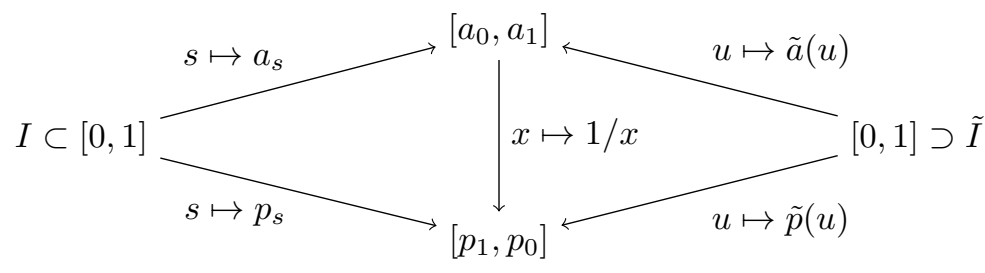

This parameter $u$ is the one given in the introduction and corresponds to a ratio of the $\gamma$, when $s$ corresponds to a ratio of $\lambda$, in the sense that:

$$
\tilde{p}\left(\frac{\gamma_{0}}{\gamma_{0}+\gamma_{1}}\right)=p\left(\frac{\lambda_{0}}{\lambda_{0}+\lambda_{1}}\right) \text {. }
$$

Remark 3.1. As already mentioned above, the parameter $u$ and the interval $\tilde{I}$ are used implicitly in Benaïm and Lobry (2016): $u$ appears in Remark 1, and the map $S$ defined at the beginning of Section 4 is given in our notation by $S(u)=p^{-1}(\tilde{p}(u))$.

Let us study the integral $\int_{p_{1}}^{p_{0}} P(x) \theta(x) d x$. Set $y=\tilde{p}^{-1}(x)$, so that:

$$
\begin{aligned}
x & =\tilde{p}(y)=\frac{1}{\tilde{a}(y)}=\frac{1}{a_{0}+\delta y}, & d x & =-\delta \tilde{p}(y)^{2} d y \\
p_{0}-x & =\delta p_{0} y \tilde{p}(y), & x-p_{1} & =\delta p_{1}(1-y) \tilde{p}(y) .
\end{aligned}
$$

Changing variables in the integral yields:

$$
\begin{array}{rl}
\int_{p_{1}}^{p_{0}} & P(x) \theta(x) d x \\
= & \int_{0}^{1} P(\tilde{p}(y))\left(\delta p_{0} y \tilde{p}(y)\right)^{\gamma_{0}-1}\left(\delta p_{1}(1-y) \tilde{p}(y)\right)^{\gamma_{1}-1} \tilde{p}(y)^{-\gamma_{0}-\gamma_{1}-1} \delta \tilde{p}(y)^{2} d y \\
\quad= & \delta^{\gamma_{0}+\gamma_{1}-1} p_{0}^{\gamma_{0}-1} p_{1}^{\gamma_{1}-1} \int_{0}^{1} P(\tilde{p}(y)) \frac{1}{\tilde{p}(y)} y^{\gamma_{0}-1}(1-y)^{\gamma_{1}-1} d y \\
= & \delta^{\gamma_{0}+\gamma_{1}-1} p_{0}^{\gamma_{0}-1} p_{1}^{\gamma_{1}-1} \int_{0}^{1} \phi(y) y^{\gamma_{0}-1}(1-y)^{\gamma_{1}-1} d y \\
= & \delta^{\gamma_{0}+\gamma_{1}-1} p_{0}^{\gamma_{0}-1} p_{1}^{\gamma_{1}-1} B(u v,(1-u) v) \mathbb{E}\left[\phi\left(U_{u, v}\right)\right]
\end{array}
$$

since $\phi(y)=\frac{1}{\tilde{p}(y)} P(\tilde{p}(y))$. A similar computation gives the exact formula

$$
C^{-1}=\left(\delta^{\gamma_{0}+\gamma_{1}-1} p_{0}^{\gamma_{0}-1} p_{1}^{\gamma_{1}-1} B(u v,(1-u) v)\right) p_{0} p_{1} \delta\left(\frac{1}{\alpha_{0}}(1-u)+\frac{1}{\alpha_{1}} u\right)
$$


for the normalization constant $C$. Therefore

$$
\Lambda_{\mathbf{y}}\left(\gamma_{0}, \gamma_{1}\right)=\frac{1}{\delta\left(\frac{1}{\alpha_{0}}(1-u)+\frac{1}{\alpha_{1}} u\right)} \mathbb{E}\left[\phi\left(U_{u, v}\right)\right] .
$$

Let us study $\phi$ more precisely. Since $P$ is a second-degree polynomial, let us write it down as $P(x)=A_{2} x^{2}+A_{1} x+A_{0}$. Then

$$
\phi(y)=\frac{A_{2}}{a_{0}+\delta y}+A_{1}+A_{0}\left(a_{0}+\delta y\right) .
$$

The second derivative is

$$
\phi^{\prime \prime}(y)=\frac{2 A_{2} \delta^{2}}{\left(a_{0}+\delta y\right)^{3}},
$$

which has the sign of $A_{2}$ on $[0,1]$, so $\phi$ is either strictly convex or strictly concave. However, the proof of the first item of Proposition 2.2 in Benaim and Lobry (2016) shows that (still in the case $\left.a_{0}<a_{1}\right) P\left(p_{s}\right)=\frac{\beta_{s}}{\alpha_{1} s}\left(1-a_{0} / a_{s}\right)\left(1-c_{s} / a_{s}\right)$ has the same sign as $a_{s}-c_{s}$, that is, $P\left(p_{s}\right)$ is positive iff $s \in I$. If $I$ is empty, so is $\tilde{I}$, and $\phi$ is negative on ]0,1[. If $I$ is not empty, so is $\tilde{I}$, and $\phi$ is positive on $\tilde{I}$ (and nonpositive outside $\tilde{I}$ ), therefore $\phi$ must be concave.

\section{Shape of the positivity region}

We begin with a lemma, which is proved in the next two sections. The existence and properties of $v_{c}$ and $t_{c}$ stated in Theorem 1.9 are deduced from this lemma in Section 4.3.

Lemma 4.1. If I is non empty, the map $(u, v) \mapsto \mathbb{E}\left[\phi\left(U_{u, v}\right)\right]$ is (strictly) increasing in $v$ and concave in $u$.

4.1. Monotonicity in $v$. We wish to compare $\mathbb{E}\left[\phi\left(U_{u, v}\right)\right]$ for different values of $v$. Since the function $\psi=(-\phi)$ is convex, a natural idea is to compare the distributions of $U_{u, v}$ for various $v$ in the convex order. Let us first recall the definition of this order.

Definition 4.2 (Convex order). Let $X$ and $Y$ be two random variables. If the inequality

$$
\mathbb{E}[\psi(X)] \leqslant \mathbb{E}[\psi(Y)]
$$

holds for all convex functions $\psi$ such that the expectations exist, $X$ is said to be smaller than $Y$ in the convex order. We denote by $X \leqslant_{\operatorname{cvx}} Y$ this relation.

The convex order admits the following characterization in terms of cumulative distribution functions (Shaked and Shanthikumar, 2007, Theorem 3.A.1).

Theorem 4.3 (Convex order and distribution functions). The variables $X$ and $Y$ satisfy $X \leqslant_{\mathrm{cvx}} Y$ if and only if $\mathbb{E}[X]=\mathbb{E}[Y]$ and, for all $x$,

$$
\int_{-\infty}^{x} F_{X}(t) d t \leqslant \int_{-\infty}^{x} F_{Y}(t) d t<\infty,
$$

where $F_{X}$ and $F_{Y}$ are the cumulative distribution functions of $X$ and $Y$. 
Remark 4.4 (Terminology). The convex order is one possible way of formalizing the idea that $Y$ is "more variable/more spread out" than $X$. Note in particular if $X \leqslant_{\mathrm{cvx}} Y$ then $\operatorname{Var}(X) \leqslant \operatorname{Var}(Y)$. For details on the convex order, a survey of other formalizations of variability and many other notions of stochastic order we refer to Shaked and Shanthikumar (2007).

Let us also note that, when (4.1) holds (without assuming $\mathbb{E}[X]=\mathbb{E}[Y]$ ), $X$ is said to second-order stochastically dominate $Y$; this is equivalent to asking the inequality $\mathbb{E}[\psi(X)] \leqslant \mathbb{E}[\psi(Y)]$ for any non-increasing convex function $\psi$. We refer to Marshall et al. (2011, Appendix B.19) for a proof, additional discussion, and references to the literature.

We will need the following easy fact.

Theorem 4.5. If $X \leqslant_{\mathrm{cvx}} Y$ and $\mathbb{E}[\psi(X)]=\mathbb{E}[\psi(Y)]$ for some strongly convex $\psi$, then $X$ and $Y$ have the same distribution.

Proof: Suppose that $X$ and $Y$ satisfy the hypotheses. By a classical characterization of the convex order (Shaked and Shanthikumar, 2007, Theorem 3.A.4) there exists a couple $(X, Z)$ such that $\mathbb{E}[Z \mid X]=0$ and $X+Z$ has the same distribution as $Y$. Since $\psi$ is strongly convex, there exists an $m>0$ such that for all $t \in[0,1]$,

$\psi(X+t Z)=\psi((1-t) X+t(X+Z)) \leqslant(1-t) \psi(X)+t \psi(X+Z)-\frac{m t(1-t)}{2} Z^{2}$.

Taking expectations we get

$$
\mathbb{E}[\psi(X)] \leqslant \mathbb{E}[\psi(X+t Z)] \leqslant(1-t) \mathbb{E}[\psi(X)]+t \mathbb{E}[\psi(Y)]-\frac{m t(1-t)}{2} \mathbb{E}\left[Z^{2}\right],
$$

where the first inequality comes from Jensen's inequality and $\mathbb{E}[Z \mid X]=0$. Since $\mathbb{E}[\psi(Y)]=\mathbb{E}[\psi(X)], Z$ must be zero almost surely, so $X$ and $Y$ have the same distribution.

Theorem 4.6 (Orderings between Beta r.v.). Let $X \sim \operatorname{Beta}(\alpha, \beta)$ and $X^{\prime} \sim$ $\operatorname{Beta}\left(\alpha^{\prime}, \beta^{\prime}\right)$. If $\alpha<\alpha^{\prime}, \beta<\beta^{\prime}$ and $\alpha /(\alpha+\beta)=\alpha^{\prime} /\left(\alpha^{\prime}+\beta^{\prime}\right)$, then $X^{\prime} \leqslant$ cvx $X$.

Proof: Call $f_{\alpha, \beta}, f_{\alpha^{\prime}, \beta^{\prime}}$ the densities of the distributions. Compute their ratio:

$$
\frac{f_{\alpha^{\prime}, \beta^{\prime}}(x)}{f_{\alpha, \beta}(x)}=C_{\alpha, \beta, \alpha^{\prime}, \beta^{\prime}} x^{\alpha^{\prime}-\alpha}(1-x)^{\beta^{\prime}-\beta} .
$$

In the first case, this ratio starts and ends in zero, is strictly increasing on $\left[0, x_{0}\right]$ and strictly decreasing on $\left[x_{0}, 1\right]$. Since the two functions are densities, the ratio must cross 1 exactly twice, say in $x_{1}, x_{2}$. Therefore

$$
d(x)=f_{\alpha^{\prime}, \beta^{\prime}}-f_{\alpha, \beta}
$$

is positive on $\left(x_{1}, x_{2}\right)$ and negative on $\left(0, x_{1}\right)$ and $\left(x_{2}, 1\right)$. Therefore

$$
D(x)=F_{X^{\prime}}(x)-F_{X}(x)
$$

starts at zero, decreases on $\left[0, x_{1}\right]$, increases on $\left[x_{1}, x_{2}\right]$ and decreases on $\left[x_{2}, 1\right]$, so $D(x)$ is negative on $\left[0, x_{3}\right]$ and positive on $\left[x_{3}, 1\right]$ (since it ends at zero). Integrating once more,

$$
\int_{0}^{x} D(t) d t
$$

starts and ends at zero (since $\mathbb{E}[X]=\mathbb{E}\left[X^{\prime}\right]$ ) and is decreasing-increasing, therefore it is non- positive. Thanks to Theorem 4.3, this implies $X^{\prime} \leqslant \mathrm{cvx} X$. 
Proof of the monotonicity in $v$ : Suppose $v<v^{\prime}$ and put $\alpha=u v, \alpha^{\prime}=u v^{\prime}, \beta=$ $(1-u) v, \beta^{\prime}=(1-u) v^{\prime}$. The theorem shows that $U_{u, v^{\prime}} \leqslant{ }_{\mathrm{cvx}} U_{u, v}$. Since $\psi=(-\phi)$ is strongly convex, this implies

$$
\mathbb{E}\left[\phi\left(U_{u, v}\right)\right]<\mathbb{E}\left[\phi\left(U_{u, v^{\prime}}\right)\right],
$$

by Theorem 4.5, so $v \mapsto \mathbb{E}\left[\phi\left(V_{u, v}\right)\right]$ is (strictly) increasing.

4.2. Concavity in $u$. Even though $\phi$ is concave and the r.v. $U_{u, v}, U_{u^{\prime}, v}$ are stochastically comparable (for the usual stochastic order), this is not enough to show the concavity of $u \mapsto \mathbb{E}\left[\phi\left(U_{u, v}\right)\right]$. We prove it by elementary means. First, recalling the explicit expression of $\phi$ we write:

$$
\begin{aligned}
\mathbb{E}\left[\phi\left(U_{u, v}\right)\right] & =A \mathbb{E}\left[\frac{1}{a_{0}+\delta U_{u, v}}\right]+B+C \mathbb{E}\left[a_{0}+\delta U_{u, v}\right] \\
& =A \mathbb{E}\left[\frac{1}{a_{0}+\delta U_{u, v}}\right]+B+C\left(a_{0}+\delta u\right) .
\end{aligned}
$$

Since $A$ is negative (see the proof of Lemma 1.8) and the last term is linear, it is enough to show that

$$
g: u \mapsto \mathbb{E}\left[\frac{1}{a_{0}+\delta U_{u, v}}\right]
$$

is convex. It is a bit easier to use the symmetry of Beta random variables $\left(U_{u, v}\right.$ and $1-U_{1-u, v}$ have the same distribution) and look at

$$
h: u \mapsto \mathbb{E}\left[\frac{1}{a_{1}-\delta U_{u, v}}\right] .
$$

Since $g(u)=h(1-u), g$ will be convex if $h$ is convex. Now, recalling that $\delta=$ $\left(a_{1}-a_{0}\right)<a_{1}$, we write the series development:

$$
h(u)=\frac{1}{a_{1}} \mathbb{E}\left[\sum_{k=0}^{\infty}\left(\frac{\delta}{a_{1}}\right)^{k} U_{u, v}^{k}\right]=\frac{1}{a_{1}} \sum_{k=0}^{\infty}\left(\frac{\delta}{a_{1}}\right)^{k} \mathbb{E}\left[U_{u, v}^{k}\right]
$$

so $h$ is a mixture of the functions $h_{k}$ given by:

$$
h_{k}(u)=\mathbb{E}\left[U_{u, v}^{k}\right]=\prod_{r=0}^{k-1} \frac{u v+r}{v+r}=\frac{1}{\prod_{r=0}^{k-1}(v+r)}(u v)(u v+1) \cdots(u v+k-1) .
$$

This is a polynomial function of $u$ with positive coefficients, therefore it is convex on $[0,1]$. This concludes the proof.

4.3. Properties of the frontier. We have just shown that $v \mapsto \tilde{\Lambda}_{\mathbf{y}}(u, v)$ is increasing. From Benaïm and Lobry (2016, Proposition 2.2) and the fact that in the change of variables $(s, t) \leftrightarrow(u, v), u$ only depends on $s$, and for fixed $u, v$ is an increasing function of $t$, we know additionally that it admits:

- negative limits at 0 and $\infty$ if $u$ does not belong to the closure of $\tilde{I}$,

- a negative limit at 0 and a positive limit at $\infty$ if $u \in \tilde{I}$.

Since $\tilde{\Lambda}_{\mathbf{y}}$ has the same sign as $\mathbb{E}\left[\phi\left(U_{u, v}\right)\right]$, this justifies the existence of $v_{c}$, and we have

$$
\tilde{\Lambda}_{\mathbf{y}}(u, v)=0 \Longleftrightarrow u \in \tilde{I}, v=v_{c}(u) .
$$

Let us prove that $v_{c}$ is quasi-convex. Let $a<b<c$ be three points in $(0,1)$. If $a$ or $c$ are not in $\tilde{I}, v_{c}(b)$ is less than $\max \left(v_{c}(a), v_{c}(c)\right)=\infty$. If $a$ and $c$ are in $\tilde{I}$, 
let $M>\max \left(v_{c}(a), v_{c}(c)\right)$. Since $\tilde{\Lambda}_{\mathbf{y}}(u, \cdot)$ is increasing, $\tilde{\Lambda}_{\mathbf{y}}(a, M)$ and $\tilde{\Lambda}_{\mathbf{y}}(c, M)$ are positive. Since $u \mapsto \mathbb{E} \phi\left(U_{u, v}\right)$ is concave, $\tilde{\Lambda}_{\mathbf{y}}(b, M)$ is positive. Therefore $v_{c}(c) \leqslant M$. Sending $M$ to $\max \left(v_{c}(a), v_{c}(b)\right)$ yields the quasi-convexity of $v_{c}$.

Let us now show the regularity properties. Let $u_{n}$ be an increasing sequence in $\tilde{I}$, converging to some $u \in(0,1)$. Since $v_{c}$ is quasi-convex, $v_{n}=v_{c}\left(u_{n}\right)$ is eventually monotone, so it converges to some $v \in[0, \infty]$. If $v$ is finite, since the zero set of $\Lambda_{\mathbf{y}}$ is closed, by continuity, $v=v_{c}(u)$, so $u$ must be in $\tilde{I}$. Conversely, if $u \in \tilde{I}, v_{c}$ is bounded on a neighborhood of $u$ by quasi-convexity, so $v$ is finite. This shows that $v_{c}$ is continuous on $\tilde{I}$ and converges to $\infty$ at the endpoints.

The properties of the change of variables $(s, t) \leftrightarrow(u, v)$ show that $v_{c}$ is welldefined and continuous with the correct limits.

\section{Support of the invariant measure}

Note that the stochastic Lotka-Volterra process has at least two invariant probability measures, supported on the coordinate axes. In the persistence regime, we are interested in the third invariant measure, whose support $\Gamma \times\{0,1\}$ is such that $\Gamma$ has non empty interior. Several properties of $\Gamma$ are established in Benaïm and Lobry (2016) (see below). In this section, we aim at providing a full description of $\Gamma$. Its shape essentially depends on the fact that $I \cap J$ is empty or not.

5.1. Persistence with "full support". In this subsection, we assume that $I \cap J$ is not empty. According to Lemma 2.1, the vector fields $F_{\mathcal{E}_{0}}$ and $F_{\mathcal{E}_{1}}$ are such that (2.1) holds. Let us denote by $\Sigma_{i}$ the intersection of $[0, \infty)^{2}$ and the unstable manifold of $\left(0,1 / d_{i}\right)$ and $\Gamma^{\prime}$ the bounded subset of $[0,+\infty)^{2}$ with border

$$
\Sigma_{1} \cup\left\{(x, 0): 1 / a_{1} \leqslant x \leqslant 1 / a_{0}\right\} \cup \Sigma_{0} \cup\left\{(0, y): 1 / d_{1} \leqslant x \leqslant 1 / d_{0}\right\} .
$$

Theorem 5.1. Suppose that $I \cap J \neq \emptyset$. Then, for any $(s, t) \in[0,1] \times(0, \infty)$ such that $\Lambda_{\mathbf{x}}(s, t)>0$ and $\Lambda_{\mathbf{y}}(s, t)>0$, then $\Gamma^{\prime}=\Gamma$.

Proof: Firstly, notice that the set $\Gamma^{\prime}$ is positively invariant for each flow since both vector fields $F_{\mathcal{E}_{0}}$ and $F_{\mathcal{E}_{1}}$ point inside $\Gamma^{\prime}$.

Pick an $s \in I \cap J$. The isoclines and the unstable manifold of the saddle point for the three environments $\mathcal{E}_{0}, \mathcal{E}_{1}$ and $\mathcal{E}_{s}$ are necessarily in the position depicted in Figure 5.3. Denote by $\Sigma_{s}$ the intersection of the unstable manifold of $\left(1 / a_{s}, 0\right)$ with the upper right quadrant.

First step: the set $\Sigma_{s}$ is contained in the support. Indeed, pick a point $(x, y)$ in the interior of the support (such a point exists by Benaïm and Lobry, 2016, Remark 6). The loop formed by the trajectories starting from $(x, y)$ with both flows (converging to $A_{1}:\left(1 / a_{1}, 0\right)$ and $A_{0}:\left(1 / a_{0}, 0\right)$ and the line segment $\left[A_{0}, A_{1}\right]$ is included in the support (by positive invariance). As a consequence, the support must contain a closed half ball centered on $A_{s}-$ let us call it $\mathcal{B}$. Now pick a point $(x, y) \in \Sigma_{s}$ : by definition its $\mathcal{E}_{s}$ flow converges for $t \rightarrow-\infty$ to $1 / a_{s}$. By continuity there exists a point in the past of $(x, y)$ which is in $\mathcal{B}$. Running the time forward again, the point $(x, y)$ must be in the support.

Second step: any point (strictly) between $\Sigma_{1}$ and $\Sigma_{s}$ is in $\Gamma$. Starting from such a point $(x, y)$, run the $\mathcal{E}_{1}$ flow in reverse time. The trajectory must cross $\Sigma_{s}$. So $(x, y)$ is in the future of a point in $\Sigma_{s} \subset \Gamma$, and $(x, y) \in \Gamma$ by positive invariance. 


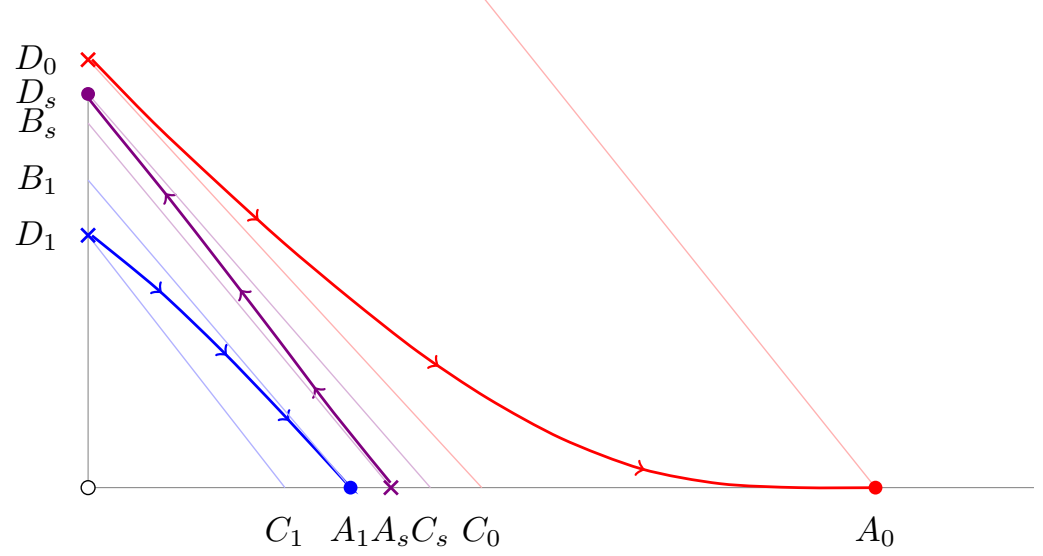

The isoclines (straight lines) and unstable manifolds (curved lines) for the three environments $\mathcal{E}_{0}$ (bottom left, in blue), $\mathcal{E}_{s}$ (middle, in purple) and $\mathcal{E}_{1}$ (upper right, in red). Note how the isoclines are "swapped" for $\mathcal{E}_{s}$, a Type 2 environment.

FiguRE 5.3. Full support case: isoclines and unstable manifolds

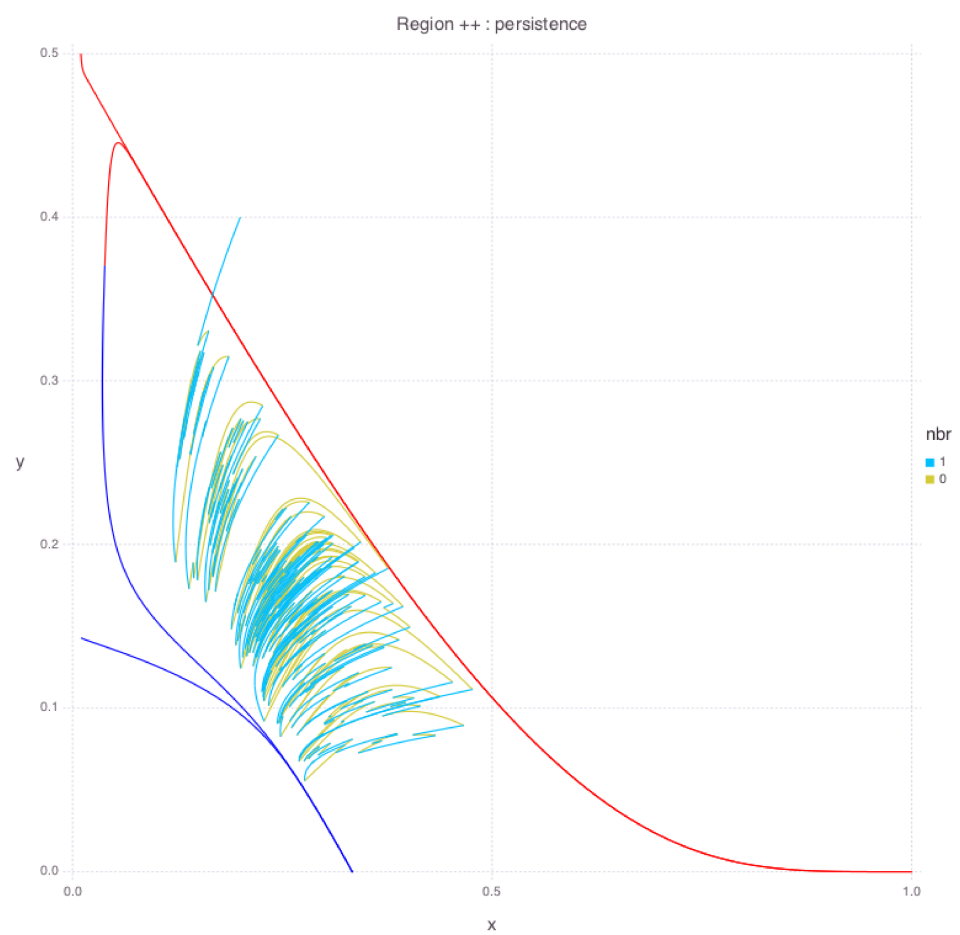

The outer curves are $\Sigma_{0}$ and $\Sigma_{1}$. The region between these curves is positively invariant. The inner curves are the two trajectories coming from the unique point $z \in T$ : they form the boundary of the support. The sample trajectory shows that the invariant measure is in practice often concentrated on a smaller subset.

FiguRE 5.4. Support away from the $y$ axis 
Third step: any point between $\Sigma_{0}$ and $\Sigma_{s}$ is in $\Gamma$. This step is similar to the previous one and is omitted.

Similarly, any point between $\Sigma_{1}$ and $\Sigma_{s}$ is in $\Gamma$.

5.2. Support away from the $y$ axis. We suppose in the sequel that $I \cap J$ is empty. Let us introduce the set where the two vector fields $F_{0}$ and $F_{1}$ are collinear:

$$
C=\left\{z \in \mathbb{R}_{+}^{2}: \operatorname{det}\left(F_{0}(z), F_{1}(z)\right)=0\right\} .
$$

This set is the union of $\{(0, y): y \geqslant 0\},\{(x, 0): x \geqslant 0\}$, and

$$
\tilde{C}=\left\{(x, y) \in \mathbb{R}_{+}^{2}: G(x, y)=0\right\}
$$

where $G$ is a polynomial of degree 2 . As a consequence, the set $\tilde{C}$ is a subset of a conic. It is easy to see that $\tilde{C}$ is also the set of non-degenerate equilibrium points for the vector field $F_{\mathcal{E}_{s}}$, as $s$ varies from 0 to 1 . When $s \in I, \mathcal{E}_{s}$ is of Type 3 so the equilibrium point is stable and globally attractive. Therefore the part of $\tilde{C}$ that corresponds to $s \in I$ must be included in $\Gamma$, as well as all trajectories (for both flows) starting from it.

Numerical experiments suggest that there is a unique "extremal point" on this part of $\tilde{C}$, such that the trajectories starting from this point form the boundary of Г. See Figure 5.4.

To describe it more precisely, consider the subset of $\tilde{C}$ made of the points where $F_{0}$ (or $\left.F_{1}\right)$ is tangent to the curve $\tilde{C}$. This set is given by

$$
T=\left\{(x, y) \in \mathbb{R}_{+}^{2}: G(x, y)=0 \text { and }\left(F_{0} \cdot \nabla G\right)(x, y)=0\right\} .
$$

Since $G$ and $F_{0} \cdot \nabla G$ are polynomials with respective degrees 2 and $3, T$ is made of at most six points according to Bezout's Theorem.

For any $z \in T$ let us define $C(z)$ the bounded region enclosed by the Jordan curve

$$
\left\{\varphi_{z}^{0}(t): t \in[0, \infty)\right\} \cup\left\{\varphi_{z}^{1}(t): t \in[0, \infty)\right\} \cup\left[1 / a_{1}, 1 / a_{0}\right] \times\{0\},
$$

where $t \mapsto \varphi_{z}^{i}(t)$ is the flow associated to the vector field $F_{i}$ for $i=0,1$.

Conjecture 5.2. The set $T$ is a singleton $\left\{z_{0}\right\}$ and the support of the invariant measure which is not supported by one of the two axes is $C\left(z_{0}\right) \times\{0,1\}$.

\section{Acknowledgements}

We thank an anonymous referee for constructive remarks. We acknowledge financial support from the French ANR project ANR-12-JS01-0006-PIECE. Numerical computations were done in Julia and graphics in TikZ.

\section{References}

M. Benaïm, S. Le Borgne, F. Malrieu and P.-A. Zitt. On the stability of planar randomly switched systems. Ann. Appl. Probab. 24 (1), 292-311 (2014). MR3161648.

M. Benaïm and C. Lobry. Lotka-Volterra with randomly fluctuating environments or "How switching between beneficial environments can make survival harder". Ann. Appl. Probab. 26 (6), 3754-3785 (2016). MR3582817. 
S. D. Lawley, J. C. Mattingly and M. C. Reed. Sensitivity to switching rates in stochastically switched ODEs. Commun. Math. Sci. 12 (7), 1343-1352 (2014). MR3210750.

A. W. Marshall, I. Olkin and B. C. Arnold. Inequalities: theory of majorization and its applications. Springer Series in Statistics. Springer, New York, second edition (2011). ISBN 978-0-387-40087-7. MR2759813.

M. Shaked and J. G. Shanthikumar. Stochastic orders. Springer Series in Statistics. Springer, New York (2007). ISBN 978-0-387-32915-4; 0-387-32915-3. MR2265633. 